VOL. 47 (1993) [205-212]

\title{
LOCALLY LIPSCHITZ FUNCTIONS ARE GENERICALLY PSEUDO-REGULAR ON SEPARABLE BANACH SPACES
}

\section{J.R. Giles and Scott Sciffer}

\begin{abstract}
For a locally Lipschitz function on a separable Banach space the set of points of Gâteaux differentiability is dense but not necessarily residual. However, the set of points where the upper Dini derivative and the Clarke derivative agree is residual. It follows immediately that the set of points of intermediate differentiability is also residual and the set of points where the function is Gateaux but not strictly differentiable is of the first category.
\end{abstract}

A real valued function $\psi$ on an open convex subset $A$ of a normed linear space $X$ is said to be locally Lipschitz on $A$ if for every $x_{0} \in A$ there exists a $K_{0}>0$ and a $\delta_{0}>0$ such that

$$
|\psi(x)-\psi(z)| \leqslant K_{0}\|x-z\| \text { for all } x, z \in A \text { and }\left\|x-x_{0}\right\|<\delta_{0} \text { and }\left\|z-x_{0}\right\|<\delta_{0}
$$

We say that $\psi$ is Gâteaux differentiable at $x \in A$ if $\lim _{\lambda \rightarrow 0}(\psi(x+\lambda y)-\psi(x)) / \lambda$ exists for all $y \in X$, denoted by $\psi^{\prime}(x)(y)$, where $\psi^{\prime}(x)$ is a continuous linear functional on $X$.

For Euclidean spaces, Rademacher [10] in 1919 gave the classical differentiability result that a real-valued Lipschitz function on an open subset is differentiable almost everywhere. In 1990, this result was spectacularly extended by Preiss [9] who proved that on a Banach space which has an equivalent norm Gâteaux differentiable away from the origin, a real-valued locally Lipschitz function on an open subset is Gâteaux differentiable on a dense subset of its domain.

For separable Banach spaces, Mazur [6] in 1933 proved that a continuous convex function on an open convex subset is Gâteaux differentiable on a dense $G_{\delta}$ subset of its domain. There has been considerable development of differentiability theory for continuous convex functions since the paper [1] by Asplund in 1968; (see the 1989 lecture notes by Phelps [8]).

However, it is well known that the extension of differentiability theory from continuous convex to locally Lipschitz functions is fraught with difficulties. In particular,

Received 4 March 1992

Copyright Clearance Centre, Inc. Serial-fee code: 0004-9729/93 \$A2.00+0.00. 
even on the real line, the set of points of differentiability of a locally Lipschitz function need not contain a dense $G_{\delta}$ subset.

For a locally Lipschitz function $\psi$ on an open subset $A$ of a normed linear space $X$, the upper Dini derivative at $x \in A$ in the direction $y \in X$,

$$
\psi^{+}(x)(y)=\limsup _{\lambda \rightarrow 0+} \frac{\psi(x+\lambda y)-\psi(x)}{\lambda}
$$

and the lower Dini derivative at $x \in A$ in the direction $y \in X$,

$$
\psi^{-}(x)(y)=\liminf _{\lambda \rightarrow 0+} \frac{\psi(x+\lambda y)-\psi(x)}{\lambda}
$$

both exist for all $y \in X$. Clearly, $\psi^{-}(x)(y)=-(-\psi)^{+}(x)(y)$, and $\psi$ has a right hand derivative at $x \in A$ in the direction $y \in X$,

$$
\psi_{+}^{\prime}(x)(y)=\lim _{\lambda \rightarrow 0+} \frac{\psi(x+\lambda y)-\psi(x)}{\lambda}
$$

if and only if $\psi^{+}(x)(y)=\psi^{-}(x)(y)$.

For a continuous convex function $\phi$ on an open convex subset $A$ of $X$, the right hand derivative $\phi_{+}^{\prime}(x)(y)$ always exists for all $x \in A$ and all $y \in X$, and given $x \in A$, $\phi_{+}^{\prime}(x)(y)$ is a continuous sublinear functional in $y$.

For a locally Lipschitz function $\psi$ on an open subset $A$ of a normed linear space $X$, given $x \in A$, the upper Dini derivative $\psi^{+}(x)(y)$ is not necessarily sublinear, but a workable extension of this derivative was devised in 1975 by Clarke, [3]. The Clarke generalised derivative at $x \in A$ in the direction $y \in X$ is defined as

$$
\psi^{0}(x)(y)=\limsup _{\substack{z \rightarrow x \\ \lambda \rightarrow 0+}} \frac{\psi(z+\lambda y)-\psi(z)}{\lambda}
$$

and given $x \in A, \psi^{0}(x)(y)$ is a continuous sublinear functional in $y$. If $\psi$ is Gâteaux differentiable at $x \in A$ and $\psi^{\prime}(x)(y)=\psi^{0}(x)(y)$ for all $y \in X$ then we say that $\psi$ is strictly differentiable at $x$. If $\psi$ is Gâteaux differentiable on a dense subset $D$ of $A$ and is strictly differentiable at $x \in A$ then the derivatives $\psi^{\prime}(z)$ are weak $*$ convergent to $\psi^{\prime}(x)$ for $z \in D$ as $z$ approaches $x$, [5]. We say that $\psi$ is regular at $x \in A$ if $\psi$ has a right hand derivative at $x$ and $\psi_{+}^{\prime}(x)(y)=\psi^{0}(x)(y)$ for all $y \in X$ and is pseudo-regular at $x \in A$ if $\psi^{+}(x)(y)=\psi^{0}(x)(y)$ for all $y \in X,[2]$. A continuous convex function $\phi$ on an open convex subset $A$ of $X$ is regular for all $x \in A$ and is strictly differentiable at $x \in A$ if and only if it is Gateaux differentiable at $x$.

In this paper we aim to prove, as a categorical extension of Mazur's Theorem for continuous convex functions, that on a separable Banach space, a locally Lipschitz 
function on an open subset is pseudo-regular on a residual subset of its domain. This will have considerable implications for other differentiability properties of locally Lipschitz functions on separable Banach spaces.

We establish our result by examining continuity properties associated with the upper Dini derivatives. For a locally Lipschitz function $\psi$ on an open subset $A$ of a normed linear space $X$ and $x \in A$ and $y \in X$, given $p \in \mathbb{N}$ we write

and

$$
\psi_{p}^{+}(x)(y) \equiv \sup _{0<\lambda<\frac{1}{p}} \frac{\psi(x+\lambda y)-\psi(x)}{\lambda}
$$

and

so that

$$
\psi_{p}^{-}(x)(y) \equiv \inf _{0<\lambda<\frac{1}{p}} \frac{\psi(x+\lambda y)-\psi(x)}{\lambda}
$$

$$
\psi^{+}(x)(y)=\lim _{p \rightarrow \infty} \psi_{p}^{+}(x)(y) \text { and } \psi^{-}(x)(y)=\lim _{p \rightarrow \infty} \psi_{p}^{-}(x)(y)
$$

Lemma . Consider a locally Lipschitz function $\psi$ on an open subset $A$ of a normed linear space $X$. Given $p \in \mathbb{N}$,

(i) for $x \in A, \psi_{p}^{+}(x)(y)$ and $\psi^{+}(x)(y)$ are continuous functions in $y$,

(ii) for $y \in X, \psi_{p}^{+}(x)(y)$ is lower semi-continuous in $x$.

Proof: Consider $x_{0} \in A$ with associated Lipschitz constants $K_{0}>0$ and $\delta_{0}>0$.

(i) For $y, z \in X$ where $\|\lambda y\|<\delta_{0}$ and $\|\lambda z\|<\delta_{0}$ we have

$$
\begin{gathered}
\left|\sup _{0<\lambda<\frac{1}{p}} \frac{\psi\left(x_{0}+\lambda y\right)-\psi\left(x_{0}\right)}{\lambda}-\sup _{0<\lambda<\frac{1}{p}} \frac{\psi\left(x_{0}+\lambda z\right)-\psi\left(x_{0}\right)}{\lambda}\right| \\
\quad \leqslant \sup _{0<\lambda<\frac{1}{p}}\left|\frac{\psi\left(x_{0}+\lambda y\right)-\psi\left(x_{0}+\lambda z\right)}{\lambda}\right| \leqslant K_{0}\|y-z\| .
\end{gathered}
$$

(ii) Given $0<\varepsilon<2 K_{0} \delta_{0}$ there exists a $0<\lambda_{0}<1 / p$ such that

$$
\frac{\psi\left(x_{0}+\lambda_{0} y\right)-\psi\left(x_{0}\right)}{\lambda_{0}}>\psi_{p}^{+}\left(x_{0}\right)(y)-\frac{\varepsilon}{2} .
$$

So for all $x \in A$ where $\left\|x-x_{0}\right\|<\min \left(\left(\varepsilon \lambda_{0}\right) /\left(4 K_{0}\right), \delta_{0}\right)$,

$$
\frac{\psi\left(x+\lambda_{0} y\right)-\psi(x)}{\lambda_{0}}>\psi_{0}^{+}\left(x_{0}\right)(y)-\varepsilon .
$$

Then $\psi_{p}^{+}(x)(y)>\psi_{p}^{+}\left(x_{0}\right)(y)-\varepsilon$. 
THEOREM. A locally Lipschitz function $\psi$ on an open subset $A$ of a separable Banach space $X$ is pseudo-regular on a dense $G_{\delta}$ subset of $A$.

Proof: For $y \in X$, given $p \in \mathbb{N}$ we have $\psi_{p}^{+}(x)(y)$ is lower semi-continuous on $A$. So there exists a dense $G_{\delta}$ subset $D_{p}^{+}$of $A$ where $\psi_{p}^{+}(x)(y)$ is continuous at each $x \in D_{p}^{+}$. The set $D_{p}^{+}=\bigcap_{p=1}^{\infty} D_{p}^{+}$is also a dense $G_{\delta}$ subset of $A$ and $\psi_{p}^{+}(x)(y)$ is continuous at each $x \in D_{y}^{+}$for every $p \in \mathbb{N}$. So for $x \in D_{y}^{+}$, given $p, q \in \mathbb{N}$ and $\varepsilon>0$ there exists a $0<\delta_{p}<1 / q$ such that

$$
\left|\psi_{p}^{+}(z)(y)-\psi_{p}^{+}(x)(y)\right|<\varepsilon \text { for all } z \in A \text { and }\|z-x\|<\delta_{p} .
$$

So

$$
\left|\sup _{\|z-x\|<\delta_{p}}\left(\sup _{0<\lambda<\frac{1}{p}} \frac{\psi(z+\lambda y)-\psi(z)}{\lambda}\right)-\psi_{p}^{+}(x)(y)\right| \leqslant \varepsilon .
$$

This holds for all $p, q \in \mathbb{N}$ so

$$
\left|\lim _{\substack{p \rightarrow \infty \\ q \rightarrow \infty}}\left(\sup _{\|z-x\|<\delta_{p}<\frac{1}{q}}\left(\sup _{0<\lambda<\frac{1}{p}} \frac{\psi(z+\lambda y)-\psi(z)}{\lambda}\right)-\psi_{p}^{+}(x)(y)\right)\right| \leqslant \varepsilon .
$$

But

so

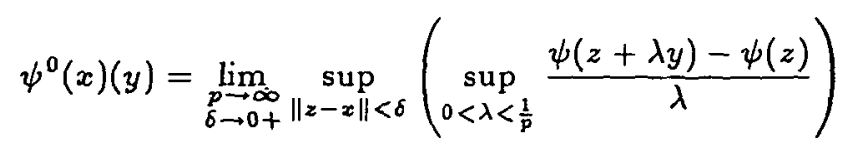

and we conclude that $\psi^{+}(x)(y)=\psi^{0}(x)(y)$.

Since $X$ is separable there exists a countable dense set $\left\{y_{n}\right\}$ in $X$. For each $n \in \mathbb{N}$ there exists a dense $G_{\delta}$ subset $D_{n}^{+}$of $A$ where $\psi_{p}^{+}(x)\left(y_{n}\right)$ is continuous at each $x \in D_{n}^{+}$. Now $D^{+} \equiv \bigcap_{n=1}^{\infty} D_{n}^{+}$is a dense $G_{\delta}$ subset of $A$ and for each $x \in D^{+}$

$$
\psi^{+}(x)\left(y_{n}\right)=\psi^{0}(x)\left(y_{n}\right) \quad \text { for all } n \in \mathbb{N} .
$$

But as both $\psi^{+}(x)(y)$ and $\psi^{0}(x)(y)$ are continuous in $y$ we conclude that for every $x \in D^{+}$

$$
\psi^{+}(x)(y)=\psi^{0}(x)(y) \quad \text { for all } y \in X .
$$

Recently, Fabian and Preiss [4] introduced the concept of intermediate differentiability. A locally Lipschitz function $\psi$ on an open subset $A$ of a normed linear space $X$ 
is said to be intermediately differentiable at $x \in A$ if there exists a continuous linear functional $f$ on $X$ such that

$$
\psi^{-}(x)(y) \leqslant f(y) \leqslant \psi^{+}(x)(y) \quad \text { for all } y \in X .
$$

They proved that, for a large class of Banach spaces, those which can be represented as a subspace of a Stegall GSG space, a locally Lipschitz function defined on an open subset of such a space is generically intermediately differentiable. Our Theorem enables us to prove their result in an elementary manner for separable Banach spaces.

Associated with the Clarke generalised derivative is the Clarke generalised subdifferential. Given $x \in A$,

$$
\partial \psi^{0}(x) \equiv\left\{f \in X^{*}: f(y) \leqslant \psi^{0}(x)(y) \text { for all } y \in X\right\} .
$$

Since $\psi^{0}(x)(y)$ is a continuous sublinear functional in $y, \partial \psi^{0}(x)$ is non-empty.

Corollary 1. A locally Lipschitz function $\psi$ on an open subset $A$ of a separable Banach space $X$ is intermediately differentiable on a dense $G_{\delta}$ of $A$.

Proof: Now $\psi$ is pseudo-regular on a dense $G_{\delta}$ subset $D^{+}$of $A$ and $(-\psi)$ is pseudo-regular on a dense $G_{\delta}$ subset $D^{-}$of $A$. For all $x \in D^{+} \cap D^{-}$a dense $G_{\sigma}$ subset of $A$, there exists a continuous linear functional $f$ on $X$ such that

$$
f(y) \leqslant \psi^{0}(x)(y) \text { for all } y \in X .
$$

So

$$
-(-\psi)^{0}(x)(y) \leqslant f(y) \leqslant \psi^{0}(x)(y) \text { for all } y \in X
$$

But for $x \in D$,

and

$$
\begin{aligned}
\psi^{+}(x)(y) & =\psi^{0}(x)(y) \\
\psi^{-}(x)(y)=-(-\psi)^{+}(x)(y) & =-(-\psi)^{0}(x)(y) \text { for all } y \in X .
\end{aligned}
$$

So

$$
\psi^{-}(x)(y) \leqslant f(y) \leqslant \psi^{+}(x)(y) \text { for all } y \in X
$$

A continuous convex function $\phi$ on an open convex subset $A$ of a normed linear space $X$ is Gâteaux differentiable at $x \in A$ if and only if it is intermediately differentiable at $x$. So Corollary 1 provides another proof of Mazur's Theorem.

Corollary 1 actually shows that, for a locally Lipschitz function $\psi$ on an open subset $A$ of a separable Banach space $X$, the set of points where $\psi$ is both pseudo-regular and intermediately differentiable is residual in $A$. 
It is well known that for a continuous function $\theta$ on the real line, the set of points where $\theta$ is differentiable but not strictly differentiable is of the first category, [12, p.138]. Our Theorem gives a corresponding result for locally Lipschitz functions on separable Banach spaces.

Corollary 2. For a locally Lipschitz function $\psi$ on an open subset $A$ of a separable Banach space $X$, the set of points where $\psi$ is Gâteaux differentiable but not strictly differentiable is of the first category in $A$.

Proof: The set $\left\{x \in A: \psi^{+}(x)(y)=\psi^{0}(x)(y)\right.$ for all $\left.y \in X\right\}$ is residual in A. So the set $\left\{x \in A\right.$ : there exists $y \in X$ such that $\left.\psi^{\prime}(x)(y) \neq \psi^{0}(x)(y)\right\} \subseteq$ $\left\{x \in A\right.$ : there exists $y \in X$ such that $\left.\psi^{+}(x)(y) \neq \psi^{0}(x)(y)\right\}$ is of the first category in $A$.

Corollary 2 extends for Gâteaux differentiability of locally Lipschitz functions on separable Banach spaces, a very general result proved by Zajicek [13] in 1987, for Fréchet differentiability.

The following example, given by Rockafeller $[11$, p.97], of a globally Lipschitz function on the real line, illustrates much of the diverse differentiability behaviour we have been discussing.

EXAMPLE. There exists a measurable set $S$ in $\mathbb{R}$ with the property that, for every non-empty open interval $I$, the sets $I \cap S$ and $I \cap(\mathbb{R} \backslash S)$ are both of positive measure. Define a function $\theta$ on $\mathbb{R}$ by

$$
\theta(x)= \begin{cases}+1 & \text { for } x \in S \\ -1 & \text { for } x \in \mathbb{R} \backslash S\end{cases}
$$

and a function $\psi$ on $\mathbb{R}$ by

$$
\psi(x)=\int_{0}^{x} \theta(t) d t .
$$

Then $\psi$ is globally Lipschitz with Lipschitz constant 1 and by Rademacher's Theorem is differentiable almost everywhere and $\psi^{\prime}(x)=\theta(x)$ for almost every $x \in \mathbb{R}$. From the properties of $S$ we have that $\partial \psi^{0}(x)=[-1,1]$ for every $x \in \mathbb{R}$, so $\psi$ is nowhere strictly differentiable. So from Corollary 2 , the set of points where $\psi$ is differentiable is of the first category. The set of points where $\psi$ is pseudo-regular is residual but is disjoint from the set of points where $\psi$ is differentiable. The set of points where $\psi$ is intermediately differentiable is residual and of full measure since it includes the set of points where $\psi$ is differentiable. However, the set of points where $\psi$ is intermediately differentiable but not differentiable is also residual but of measure zero.

Our results can be applied to give information about the differentiability of distance functions on separable Banach spaces. A non-empty closed set $K$ in a normed linear 
space $X$ is said to be proximinal if to each $x \in X \backslash K$ there exists a $p(x) \in K$ such that

$$
d(x) \equiv d(x, K)=\|x-p(x)\| .
$$

APPLICATION. Given a non-empty proximinal set $K$ in a separable Banach space with norm Gâteaux differentiable away from the origin, the distance function $d$ generated by $K$ is strictly differentiable on a dense $G_{\delta}$ subset of $X \backslash K$.

Proof: The distance function $d$ is a Lipschitz function so from the Theorem, we have that the set of points in $X \backslash K$ where $d$ is pseudo-regular is residual in $X \backslash K$. At such a point $x \in X \backslash K$ we have

$$
\begin{aligned}
d^{0}(x)(y) & =d^{+}(x)(y) \equiv \limsup _{\lambda \rightarrow 0+} \frac{d(x+\lambda y)-d(x)}{\lambda} \\
& \leqslant \limsup _{\lambda \rightarrow 0+} \frac{\|x-p(x)+\lambda y\|-\|x-p(x)\|}{\lambda} \\
& =\|x-p(x)\|^{\prime}(y) \text { for all } y \in X .
\end{aligned}
$$

Since $d^{0}(x)(y)$ is sublinear in $y$ we conclude that

$$
d^{0}(x)(y)=\|x-p(x)\|^{\prime}(y) \text { for all } y \in X
$$

and so $d$ is strictly differentiable at $x$.

REMARKS. 1. For a locally Lipschitz function $\psi$ on an open subset $A$ of a separable Banach space $X$, given $p \in \mathbb{N}, \psi_{p}^{+}(x)(y)$ is continuous at points $x \in D^{+}$for every $y \in X$. But this does not necessarily imply that $\psi^{+}(x)(y)$ is continuous at any point $x \in A$ for every $y \in X$. The points $x \in D^{+}$are points where $\psi$ is pseudo-regular, but a point $x \in A$ where $\psi^{+}(x)(y)$ is continuous at $x$ for every $y \in X$ is a point where $\psi$ is strictly differentiable. The former set is residual but in the Example the latter is empty.

2. We might well ask how our Theorem could be extended for a wider class of Banach spaces than the separable spaces. For a locally Lipschitz function $\psi$ on an open subset $A$ of a Banach space $X$, given $p \in \mathbb{N}, \psi_{p}^{+}(x)(y)$ is jointly lower semi-continuous on $A \times X$. So there exists a dense $G_{\delta}$ subset of $A \times X$ at the points of which $\psi_{p}^{+}(x)(y)$ is continuous in both variables. For any Banach space where we could guarantee that such a $G_{\delta}$ subset of $A \times X$ contained a subset $D \times X$ where $D$ is a $G_{\delta}$ subset of $A$, the Theorem would extend to such a space. However, such an investigation would take us beyond the problem so deftly treated by Namioka [7]. 


\section{REFERENCES}

[1] E. Asplund, 'Fréchet differentiability of convex function', Acta Math. 121 (1968), 31-47.

[2] J.M. Borwein, 'Minimal cuscos and subgradients of Lipschitz functions', in Fixed point theory and its applications, Pitman Research Notes, 252, 1991, pp. 57-81.

[3] F.H. Clarke, 'Generalized gradients and applications', Trans. Amer. Math. Soc. 205 (1975), 247-262.

[4] M. Fabian and D. Preiss, 'On intermediate differentiability of Lipschitz functions on certain Banach spaces', Proc. Amer. Math. Soc. 113 (1991), 733-740.

[5] J.R. Giles and Scott Sciffer, 'Continuity characterisations of differentiability of locally Lipschitz functions', Bull. Austral. Math. Soc. 41 (1990), 371-380.

[6] S. Mazur, 'Über schwache Konvergenz in den Raümen $\left(L^{p}\right)$ ', Studia Math. 4 (1933), 128-133.

[7] I. Namioka, 'Separate continuity and joint continuity', Pacific J. Math. 51 (1974), 515-531.

[8] R.R. Phelps, Convex functions, monotone operators and differentiability, Lecture Notes in Mathematics 1364 (Springer-Verlag, Berlin, Heidenberg, New York, 1989).

[9] D. Preiss, 'Differentiability of Lipschitz functions on Banach spaces', J. Funct. Anal. 91 (1990), 312-345.

[10] H. Rademacher, 'Über partielle und total Differenzierbarkeit von Funktionen mehrerer Variabeln und über die Transformation der Doppelintegrale', Math. Ann. 79 (1919), 340-359.

[11] R.T. Rockafeller, The theory of subgradients and its applications to problems of optimization. Convex and non-convex functions (Heldermann-Verlag, Berlin, 1981).

[12] B.S. Thomson, Real functions, Lecture Notes in Mathematics 1170 (Springer-Verlag, Berlin, Heidelberg, New York, 1985).

[13] L. Zajiček, 'Strict differentiability via differentiability', Acta Univ. Carolin. 28 (1987), 157-159.

Department of Mathematics

The University of Newcastle

Newcastle NSW 2308

Australia 